\title{
The Potential of Biomimetic Electrospun-Nanofibrous Scaffolds for Bone Tissue Engineering
}

\author{
Ha Na Park ${ }^{1}$, Jung Bok Lee ${ }^{1}$, Ho-Jin Moon ${ }^{1}$, \\ Dae Hyeok Yang ${ }^{1}$ and Il Keun Kwon ${ }^{1,2}$ \\ ${ }^{1}$ Department of Maxillofacial Biomedical Engineering, School of Dentistry \\ Institute of Oral Biology, School of Dentistry \\ Kyung Hee University, Seoul, \\ Republic of Korea
}

\section{Introduction}

Bone is a dynamic organ that is constantly being remodeled to maintain a healthy skeleton. Bone preserves skeletal composition, structural integrity and regulates mineral homeostasis for a lifetime. Bone has three important functions: first, it provides support and sites of attachment for muscles, secondly, it protects vital organs such as bone marrow and brain, and it acts as a metabolic organ with major reserves of calcium and phosphate.(Feng \& McDonald, 2011) $)^{1}$ Bone tissue is a mineralized organic structure mostly composed of collagenous fibers and calcium phosphate in the form of hydroxyapatite (HAp), with embedded cell components like osteoblasts and osteoclasts. For bone tissue regeneration, there are many studies on bone tissue regeneration with unlimited application and without the risk of disease transmission using the tissue-engineered scaffolds. There are many natural (collagen, gelatin, chitosan, and hyaluronic acid), synthetic biodegradable [poly( $\mathrm{a}-$ hydroxyacids), poly(glycolic acid) (PGA), poly(lactic acid) (PLA), and poly(lactic acid-coglycolic acid) (PLGA)] polymers, and bioceramics (bioglass, tri-calcium phosphate, and hydroxyapatite). To design scaffolds for bone regeneration, osteoconductive and osteoinductive properties, as well as non-cytotoxicity and osteogenic differentiation ambience, must be provided. Among various fabrication methods for tissue engineering scaffolds, electrospinning (ELSP) has been used to produce nonwoven, porous, nano to micro scale fibrous scaffolds. A range of novel materials and processing tools have been developed to mimic the native extracellular matrix (ECM) for potential applications as tissue engineering scaffolds and ultimately to restore degenerated functions of the tissue. These electrospun-nanofibers have attracted considerable interest in tissue regeneration, including bone reconstruction. In order to provide some kind of function to the nanofibers, drugs, peptides, or growth factors have been loaded into the sheets. Recently, hybrid/composite nanofibers using biodegradable polymers, bioactive inorganic materials that could supply suitable mechanical properties and bioactives for progenitor/stem cells have been produced.(figure 1) (Seong et al., 2010)2 

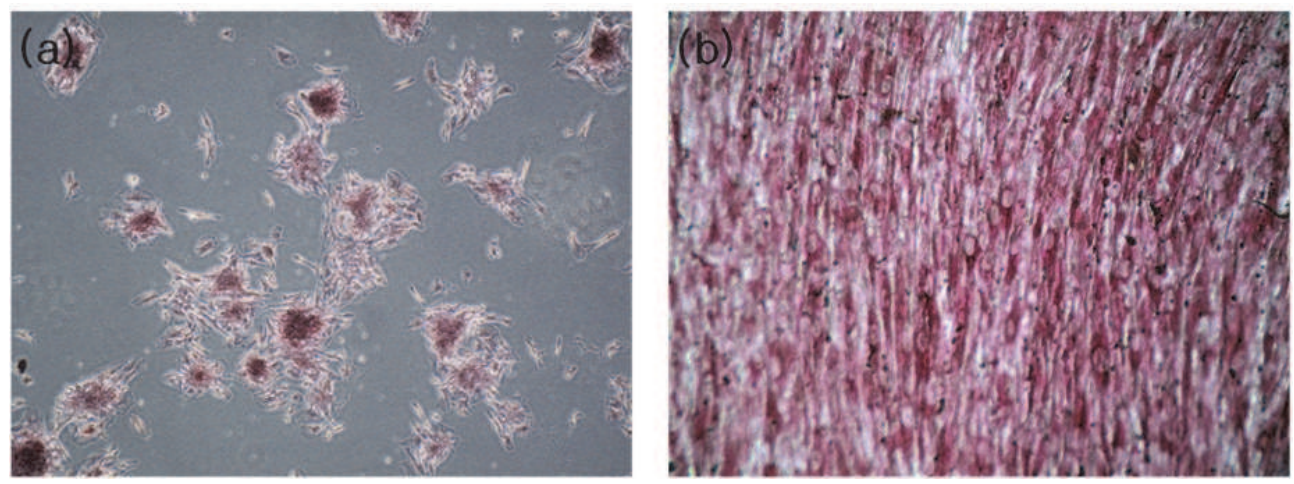

Fig. 1. Osteogenic differentiation of (a) dental pulp stem cells, and (b) adipose derived stem cells.

The nanofibers surface-functionalized with bone minerals (i.e. $\beta$-tricalcium phosphate, hydroxyapatite) or proteins/peptides are also a promising strategy for achieving therapeutic functions. Recent attempts to endow a 3D scaffolding technique to the electrospinning regime have shown some promise for engineering $3 \mathrm{D}$ tissue constructs. With the improvement in knowledge and techniques of bone-targeted nanofibrous matrices, bone tissue engineering is expected to be realized in the near future. In this chapter, we address the functionalization of electrospun-nanofibers with drugs, proteins/peptides, and growth factors, with particular focus on bone tissue regeneration. Some aspects of nanofiber design including architectural properties, surface functionalization and bio-functional materials selection are also addressed.

Electrospinning is an interesting and potentially profitable research area that provides outstanding opportunities for tissue regeneration, with the aim of repairing or replacing diseased or damaged tissue in the human body. Many studies have been done to better understand bone tissue trauma and diseases that result in disability and other problems. Therapeutic repair of skeletal tissues by electrospinning has raised great attention.

The repair of bone defects using artificial agents, which includes bone components and bone-associated cells, should be directed towards ensuring native bone recognizes and responds appropriately to form structural components.

\section{Bone remodeling}

Bone remodeling is the predominant metabolic process regulating bone structure and function. Bone remodeling involves the removal of old or damaged bone by osteoclasts (bone resorption) and the subsequent replacement of new bone formed by osteoblasts and is necessary to repair damaged bone and to maintain mineral homeostasis. The basic multicellular unit (BMU) which is known as the functional and anatomic site for bone remodeling carries this process out using various bone-lining cells, osteocytes, osteoclasts, and osteoblasts. (Parfitt, 1994; Seeman, 2009) 3 Across the BMU is a canopy of cells that creates a bone-remodeling compartment. The remodeling process involves four major distinct but overlapping phases. (a) The first phase of bone remodeling involves detection of an initiating remodeling signal. This signal can take direct mechanical strain for structural damage or hormone (estrogen or PTH) for homeostasis change. Then, in each specific site, 
activation of bone remodeling begins. (b) After activation of BMU, bone resorption is started by osteoclasts. Osteoblasts respond to signals generated by osteocytes or direct endocrine activation signals and recruit osteoclast precursors to the remodeling site. In this process, stromal-derived factors stimulate and this system allowed for production of two hematopoietic factors that are both necessary and sufficient for osteoclastogenesis. These are the TNF-related cytokine the receptor activator of nuclear factor $\mathrm{\kappa B}(\mathrm{NF}-\mathrm{\kappa B})$ ligand (RANKL) and the monocyte/macrophage colony-stimulating factor (M-CSF) (Yasuda et al., 1998; Lacey et al., 1998) ${ }^{4}$ and for the subsequent activation of RANK on the surface of hematopoietic precursor cells. (Nakagawa et al., 1998; Hsu et al., 1999) ${ }^{5}$ Together, M-CSF and RANKL are required to induce expression of genes that typify the osteoclast lineage, including those encoding tartrate-resistant acid phosphatase (TRAP) leading to the development of mature osteoclasts. Then, reversal cells follow the osteoclasts, covering the newly exposed bone surface, and prepare it for deposition of replacement bone. Subsequently macrophages produce MMPs, the enzymes required for matrix degradation, and perform phagocytosis. (Newby et al., 2008) ${ }^{6}$ (c) The remodeling process enters the next phase as osteoblast function begins to overtake bone resorption as the predominant event. Osteoblasts occupy the tail portion of the BMU by trenching the bone surface and deposit un-mineralized bone matrix known as osteoid and direct its formation and mineralization into mature lamellar bone. Osteoblast formation and function continue even after cessation of bone resorption to ensure a balance between bone removal and bone formation. (d) In the last phase, the osteoid becomes mineralized and this concludes the bone-remodeling cycle. Following mineralization mature osteoblasts go through apoptosis, return to a resting state with bone-lining cells or become embedded in the mineralized matrix, and differentiate into osteocytes. Therefore, the environment of bone surface is reestablished and maintained until the next bone remodeling process is initiated.

\section{Nanofibrous scaffold using various polymers by electrospinning}

Different kinds of materials such as synthetic materials, natural materials, and synthetic and natural blends, demonstrate a variety of mechanical properties, degradation rates, cellmaterial interactions, and biofunctions. In order to select the most proper characteristics for these polymers one must consider each material's suitability for bio-functionalization for use as bone tissue engineering scaffolds

\subsection{ELSP using synthetic polymers}

Synthetic materials have been commonly used in fabrication of fibrous sheets using electrospinning methods because of their good mechanical properties, ease of processing, and cost efficiency. Among the various synthetic materials, a family of poly(a-hydroxyl acid), polyester-based polymers, and their co-polymers, have been widely investigated due to their biodegradability, tissue integration effect, and easily controlled properties.(Yun et al., 2009; Seyedjafari et al., 2010; Haslauer et al., 2011; Lee et al., 2011; Burg et al., 2000)7

Yoshimoto et al. developed biodegradable PCL nanofiber scaffolds for bone tissue engineering. In this study the PCL scaffolds could regenerate specific defects and allowed sufficient cell penetration. Their study has shown that electrospun PCL scaffolds provide useful clinical application including an environment that supports mineralized tissue formation and may be a suitable candidate for the treatment of bone defects.( Yoshimoto et al., 2003) ${ }^{8}$ Since Yoshimoto et al. investigation of PCL, several studies that use PCL for 
bone tissue engineering have been done. (Erisken et al., 2008; Fujihara et al., 2005; Seol et al., 2010; Kolambkar et al., 2010)9 Kolambkar et al. investigated the attachment, colonization, and osteogenic differentiation of two stem cell types, human mesenchymal stem cells (hMSCs) and human amniotic fluid stem (hAFS) cells, on electrospun PCL nanofibers. (Figure 2) They seeded cells on PCL nanofiber meshes and wrapped these around a threedimensional (3D) collagen scaffold followed by 2 weeks of incubation in vitro. As a control, cells were seeded throughout the collagen scaffold alone. The collagen scaffold with cells seeded throughout had more cells on the exterior with numerous dead cells in the interior. When a cell-seeded mesh was wrapped around the scaffold, cells migrated on to the peripheral surface of the scaffold and displayed high viability. In addition cells colonized the top surface of the scaffold and migrated more than $500 \mu \mathrm{m}$ into the scaffold from the mesh. This study provides support for the use of the PCL nanofiber mesh as a scaffold for cell culture in vitro, and a cell delivery agent for the repair of bone traumatic injury. (Kolambkar et al., 2010) ${ }^{10}$

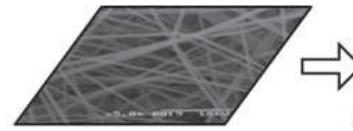

Seed cells on mesh

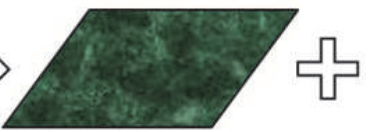

Cell attachment

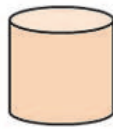

Collagen scaffold

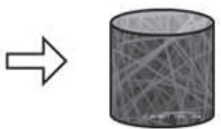

Wrap mesh

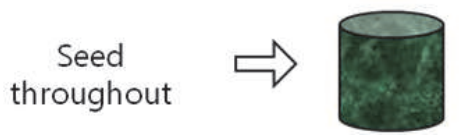

Fig. 2. Cell-seeded on nanofiber meshes to investigate the use of nanofiber meshes, cells were seeded on nanofiber meshes and wrapped around a three-dimensional (3D) collagen scaffold for 2 weeks in vitro.

Cho et al. studied the effect of hydrophilized PCL nanofiber mesh for guided bone regeneration (GBR). They fabricated a hybrid porous biodegradable membrane using PLGA membrane and electrospun PCL nanofiber mesh, PCL/PLGA hybrid membrane was compared with hydrophilized PLGA membrane, hydrophilized PCL nanofiber mesh and a commercialized GBR membrane for tensile and suture pullout strengths, model nutrient permeability, and bone regeneration behavior. The PCL/PLGA hybrid membrane showed the highest tensile and suture pullout strengths under both dry and wet conditions. In addition to these results, in vivo testing was performed and histology was done including H\&E and MT staining, the fibrous connective tissues were filled into the bone defect and new bone formation was inhibited in the blank control group. However, in the membrane groups, the new bone was continuously grown, and the PCL/PLGA hybrid membrane showed compact and faster bone regeneration than other membrane groups, even though their permeability of nutrients was higher.(Figure 3,4) (Cho et al., 2009)11

Badami et al. studied the behaviors of MC3T3-E1 osteoblastic cells seeded on a variety of electrospun synthetic fibers including spreading, proliferation, and differentiation of 

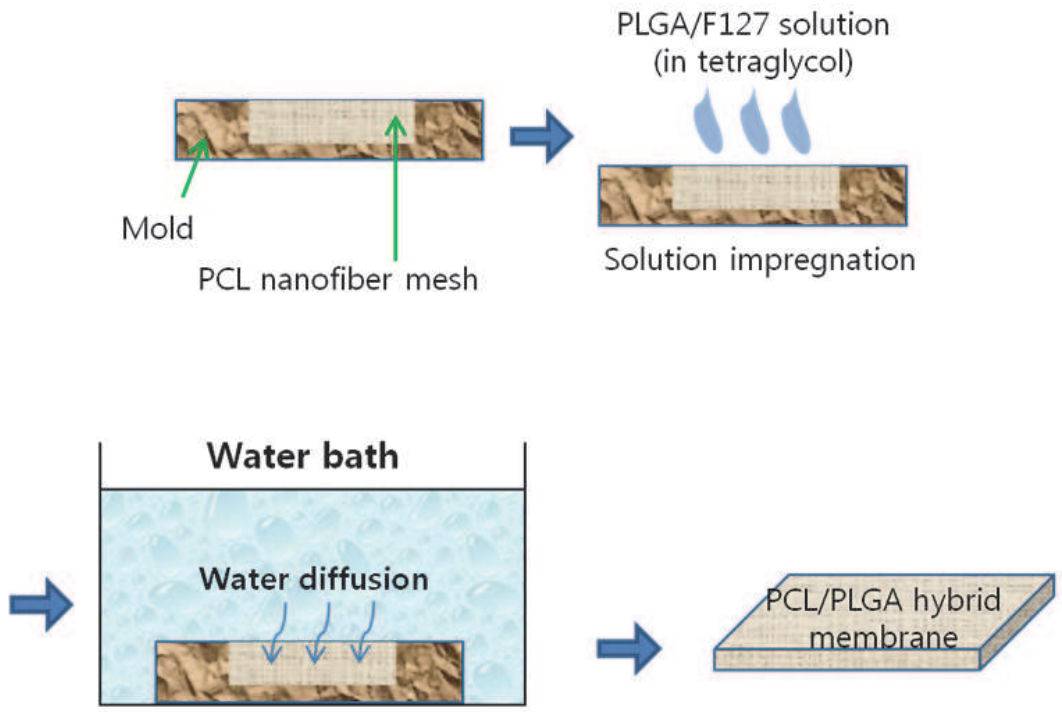

Immersion \& preciptaion (solvent/non-solvent exchange) Solidified PLGA/F127 Membrane formation

Fig. 3. Schematic of PCL/PLGA hybrid membrane fabrication process by an immersion precipitation method.

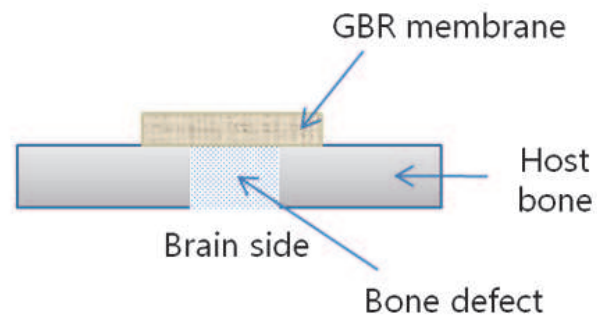

Fig. 4. Illustration of histological schematic.

MC3T3-E1. This behavior was found to depend on the fiber diameter. PLLA, PDLLA, PEGPLLA, and PEG-PDLLA were electrospun and spin-coated on glass cover slips. The density of cultured cells on the fibrous sheet was equal to or greater than that on the smooth surface membranes in the presence of osteogenic factors. These researchers mentioned that surface morphology of the designed scaffold can promote spreading, orientation, and proliferation of osteoblast-like cells. (Badami et al., 2006)12

Aligned and randomly formed elastomeric poly(ester urethane)ureas (PEUURs) fibers were electrospun, and compared with spin-coated films by Bashur et al. They synthesized PEUUR by using PCL as the soft segment and a combination of urethane and urea as the 
hard segment. Then, bone marrow stromal cells (BMSCs) were cultured on electrospun PEUUR meshes to determine the effect of fiber diameter and alignment on cell morphology, proliferation, and ligament gene expression. They demonstrated that these meshes support attachment and spreading of BMSCs. In addition, cell morphology was sensitive to fiber diameter and alignment, and adherent cells oriented parallel to fibers on aligned fiber PEUURs meshes. These results also showed that larger fibers suppressed expression of collagen a1, decorin, and tenomodulin and suggested that aligned electrospun submicron PEUURs fiber meshes may be preferable for ligament like tissue engineering. (Bashur et al., 2009) 13

Synthetic materials have been widely used in electrospinning due to their easier to control pore size, fiber diameter, structural morphology, and better mechanical properties as compared to natural materials..

\subsection{ELSP using natural materials}

The most favorable advantage of natural polymers is their inherent biocompatibility established through the presence of specific cell recognition sites that are capable of binding cells. (Haslauer et al., 2011) ${ }^{14}$ Natural polymers including collagen, silk, chitin, and their derivatives are the most popular materials for use as electrospun scaffolds. Collagen is one of the major extracellular matrix (ECM) morphologic components and bone tissue contains high levels of collagen type I. It is easily degraded and resorbed in the body and promotes cell adhesion. Silk is considered to be the most promising natural protein-type replacement for collagen in bone tissue engineering because of its biocompatibility, low degradation rate, and excellent mechanical strength. Chitosan possesses a number of useful characteristics such as structural similarity to glycosaminoglycan, improved bone formation, osteoconductivity, excellent biocompatibility, biodegradability, low immune response and good mechanical properties. However pure chitosan is difficult to electrospin due to its limited solubility, high viscosity, dielectric constant, and three-dimensional networks of strong hydrogen bonds. (Haslauer et al., 2011; Lee et al., 2011; O'Brien et al., 2004; Matthews et al., 2002; Beachley et al., 2010) ${ }^{15}$ Silk fibroin (SF) has proven to be effective in biomedical applications because it has good oxygen and water vapor permeability, and biodegradability. Kim et al. fabricated SF fibrous membranes by the electrospinning method and evaluated their biocompatibility and its effect on bone regeneration using MC-3T3 cells. This studied found that the MC-3T3 cells were well attached and proliferated on the SF membrane. They also examined the activity of alkaline phosphatase (ALP) and calcification in vitro and in vivo. These results suggested that electrospun SF possessed good biocompatibility and lead to enhanced bone regeneration without any inflammatory reaction. (Kim et al., 2005) ${ }^{16}$

\section{Functionalized fibrous sheets}

In order to give biofunction to scaffolds, biodegradable polymers have been developed with various additives such as tricalcium phosphate (TCP), hydroxyapatite (HAp), calcium composite, carbon nanotubes (CNT), natural polymers, and proteins homogeneously incorporated into the scaffold.

\subsection{Co-ELSP with natural polymer}

Synthetic polymers such as polyester-based membranes are biodegradable, allow tissue integration and are easily controlled in terms of their physical and chemical properties. 
However, they have poor cell response and bioactivity. In the case of natural polymers, they have good biocompatibility and biological activity. For these reasons, synthetic and natural polymer hybrid scaffolds have been investigated. (Haslauer et al., 2011; Lee et al., 2011; Bottino et al., 2011) ${ }^{17}$

For example, Haslauer et al. fabricated PCL fibers that were fully coated with collagen as formed by a core-sheath fiber. They examined the differentiation behavior of human adipose-derived stem cells (hASCs) on PCL/collagen sheath-core biocomponent fibrous scaffolds by proliferation, live/dead, and calcium quantity measurements. After two weeks of culture of hASCs on nanofibers, the results showed that viability, proliferation and osteogenesis of hASCs were promoted more thoroughly than on the PCL control nanofiber.(Figure 5) (Haslauer et al., 2011)18.

(A)

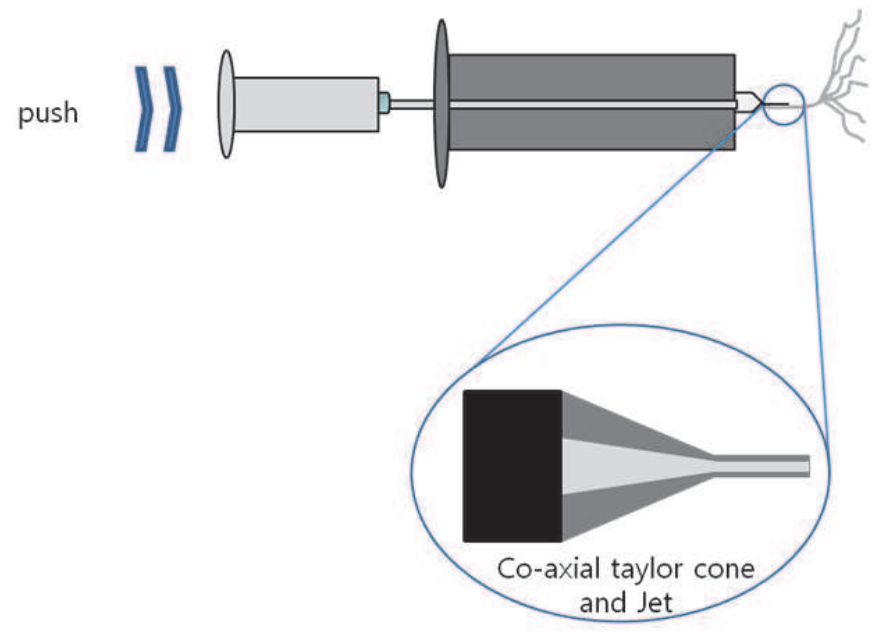

(B)

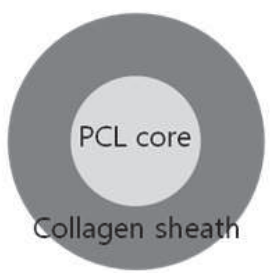

Fig. 5. Schematic illustration of core-sheath electrospinning system and its result fiber morphology.

\subsection{ELSP with inorganic particles}

In order to mimic natural ECM and bone component, or improve the mechanical properties of scaffolds, recent researchers have studied composites made with various particles including $\mathrm{HAp}, \mathrm{Ca}^{2+}$, and so on. Among various inorganic materials, $\mathrm{HAp}, \mathrm{Ca}^{2+}$ composites and $\beta$-TCP were used in research for bone regeneration because those are the major components of bone. (Erisken et al., 2008; Fujihara et al., 2005; Seol et al., 2009; Ko et al., 2008; Seol et al., 2010) ${ }^{19}$ HAp embedded in a collagen matrix has been extensively studied, 
since these are the cardinal elements of natural bone. The polymer nanofibers blended with HAp or $\beta$-TCP were able to bind to bone tissue easily because they have a similar chemical structure to the organic components of natural bone tissue. Many researchers have concluded that collagen and HAp composites are principal constituents for bone tissue engineering. (Seyedjafari et al., 2010; Nie et al., 2007; Mouthuy et al., 2010) ${ }^{20}$ Many researchers used these particles either during electrospinning or incorporated them after electrospinning.

\subsubsection{Composites made with components of natural bone}

The combination of enhanced mechanical properties, biocompatibilities, and fibrous formabilities of the developed scaffolds is believed to have great potential for various biomedical engineering applications including bone tissue regeneration. Among various inorganic materials, HAp has been widely investigated due to its similarity to the mineral component of natural bone. This is considered an osteoconductive material allowing new bone regeneration. HAp has been widely applied in biomaterials as either a coating or filling material for bone tissue engineering. Electrospun fibers are hypothesized to play a role in sustaining mechanical properties, as well as allowing for biodegradability, and acting as an actual osteoconductive scaffold after being coated by hydroxyapatite.(Figure 6) (Nie et al., 2007; Mouthuy et al., 2010; Peng et al., 2011221

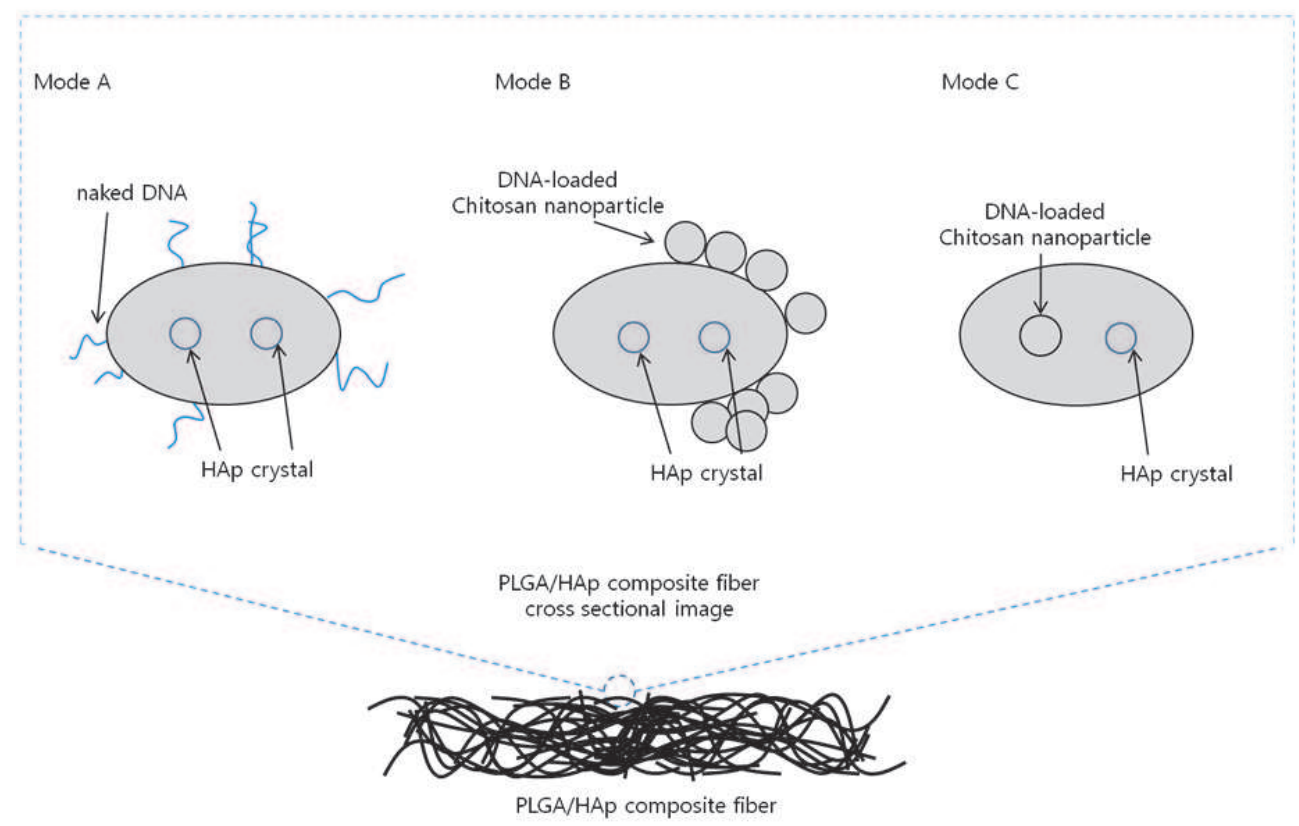

Fig. 6. Three DNA incorporation modes. (Mode A) PLGA/HAp composite fiber with naked DNA coated outside, (Mode B) PLGA/HAp composite fiber with DNA-loaded chitosan nanoparticle coated outside, and (Mode C) PLGA/HAp composite fiber with DNA-loaded chitosan nanoparticles encapsulated inside. 
Bottino et al. designed and fabricated such a system via sequential multi-layering of scaffolds. They fabricated functionally graded membrane (FGM) consisting of a core layer (CL) and both functional surface layers (SLs). CL was consisted of three layers which were different ratios of PLCL, PLA, and gelatin. And SLs were layers of PLA, gelatin, and functional factors such as HAp or metronidazole benzoate (MET). MET was commonly used as an antibiotic in the treatment of periodontitis. This FGM has enhanced function including mechanical integrity, biodegradability and cell-membrane interactions. The multilayered electrospinning method could be interesting technique for generating the more predictable characteristics that can ultimately lead to promoted regeneration. (Figure 7) (Bottino et al., 2011) 22

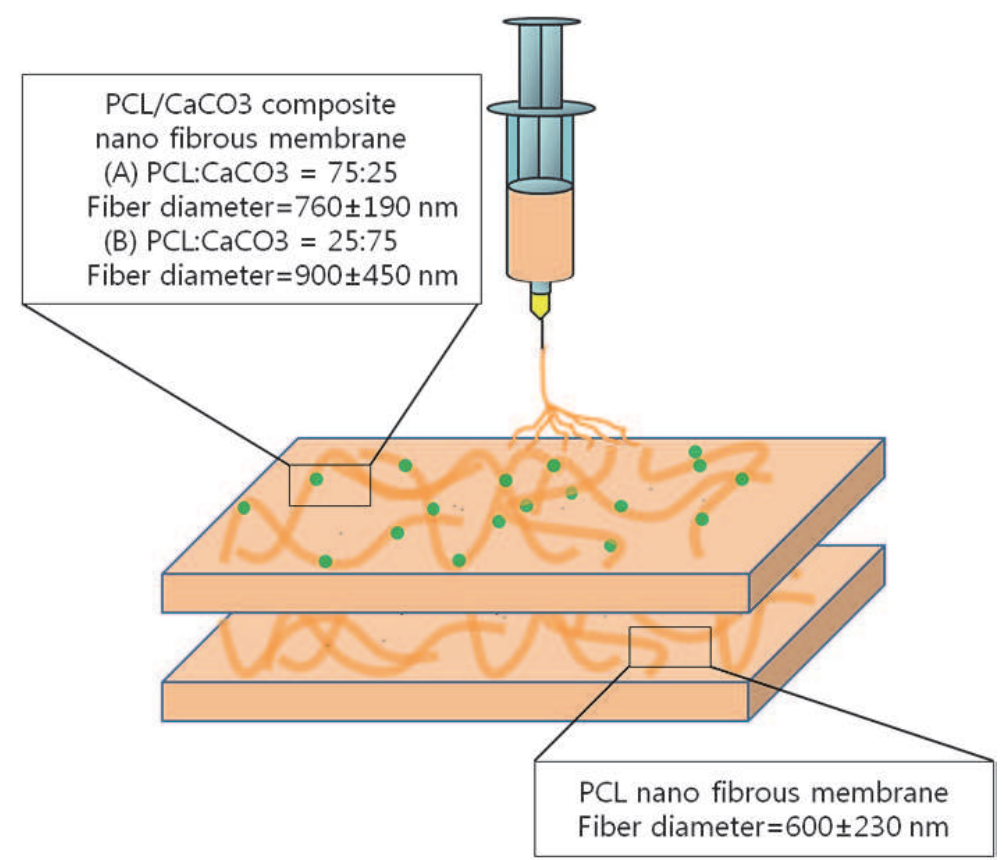

Fig. 7. Double-layered nano-fibrous GBR membranes to avoid rupture during cell seeding procedure. Two different composite membranes (i.e. (A) PCL: $\mathrm{CaCO}_{3}=75: 25$ and (B) PCL: $\left.\mathrm{CaCO}_{3}=25: 75\right)$ were prepared for osteoblast seeding.

PLLA and HAp composite scaffolds have been used widely for healing bone trauma and tissue regeneration. Seyedjafari et al. prepared PLLA nanofibrous scaffolds and deposited HAp nanoparticles (n-HAp) on the surface of these nanofibers. They investigated the capacity of n-HAp coated scaffolds for osteogenic induction using human cord blood derived unrestricted somatic stem cells (USSCs) for in vitro biocompatibility tests and subcutaneous implantation for in vivo animal tests. According to their results, these scaffolds had suitable mechanical properties for bone tissue engineering scaffolds and enhanced adhesion, proliferation, and osteogenic differentiation of USSCs. (Seyedjafari et al., 2010) ${ }^{23}$

PLLA fibrous scaffolds were also used by Whited et al. At first, they fabricated dual polymer composition scaffolds using PEO and PLLA. And then, PEO fibers were removed 
to increase pore size and porosity of the scaffolds effectively while coating the surface with a biomimetic apatite substrate. These scaffolds supported MC3T3-E1, pre-osteoblast proliferation, and osteogenic differentiation while accelerating cell infiltration into the scaffold and enhancing overall cell distribution. These results indicated that such a scaffold may facilitate enhanced osteoprogenitor maturation and colonization in vitro.(Whited et al., 2011) 24

Other groups used PLLA for preparing nanofibers and studied the potential for use as bone tissue engineering scaffolds.

Rainer et al. studied about sternal bone healing using a HAp-functionalized electrospun scaffold. They developed PLLA/HAp nanofiber by electrospinning. The surface of the nanofiber showed that HAp particles were revealed onto the fiber and an intrinsic porosity with a uniform pore size was approximately $100 \mathrm{~nm}$. They tested the ability of this previously characterized scaffold to promote bone repair in a fractured animal model. The bone marrow of a rabbit was exposed, which is one of mesenchymal bone progenitors, in order to enable a direct interaction of the endogenous cells with a scaffold actively emanating a biological osteoinductive signaling. They then characterized the healing using axial reformatted images for treated and control groups at different time points and calculated bone density (BD) at each time point. They appropriately evaluated the major outcomes of their study by means of instrumental analysis and by virtue of the reported diagnostic accuracy of radiological imaging in sternal healing process which allowed for direct measurements of mineralization and fracture rim closure. They proposed the development of a cell-free system able to exploit and boost endogenous repair and stem cell resources, guiding them towards tissue restoration, because the use of autologous cells would present an additional limit in terms of clinical application. (Rainer et al., 2011) ${ }^{25}$

Some research groups have synthesized aligned nanofiber scaffolds within n-HAp particles using a metathesis method. Preliminary research was studied by Jose et al. They fabricated fibrous scaffolds based on PLGA and n-HAp and analyzed its mechanical properties. They dispersed the appropriate nanoparticles in the PLGA solution according to the result of their degradation tests. The fiber diameter was increased with the different amounts of n-HAp (1, 5, 10 and $20 \mathrm{wt}$.\%). In this study, the increasing n-HAp concentration occurred along with an increase in the glass transition temperature. The presence of well-dispersed nano HAp particles reduced the chain mobility hence it could help to prevent shrinkage to some degree. It showed a sinusoidal trend with a slight decrease in modulus by a week due to the plasticizing effect of the medium which was followed by an increase because shrinkage, and a subsequent drop by 6 th week due to degradation. (Jose et al., 2009)26

Yun et al. attempted to characterize the gene expression pattern during osteogenic differentiation of various stem cells on PLGA and HAp biocomposite nanofibrous scaffolds. PLGA and HAp biocomposite nanofibrous scaffolds were fabricated by electrospinning at a 5:1 blending ratio. They cultured primary adipose tissue-derived stem cells (hADSCs), bone marrow cells (MSCs), and dental pulp cells (PCs) from human in order to evaluate the biocompatibility of these scaffolds. Cytotoxicity of the scaffolds were tested using NIH-3T3 fibroblast cells. To regenerate new bone tissues, several biological properties are required for these scaffolds. Improved differentiation into osteogenic cells also improves the opportunity for bone and tooth regeneration and, continuous differentiations of stem cells into osteogenic cells were comparative investigated on this scaffold.(Yun et al., 2009)27 
Peng et al. prepared HAp/PLLA random or aligned fibrous scaffolds and both types of HAp particles were oriented well along the long axes of fiber. The influence of the scaffold composition, HAp particle size (nano or micro) and fibrous morphology (aligned or randomized) were discussed. All of the HAp/PLLA scaffolds exhibited good biocompatibility and cell signaling property and better support for cell attachment, proliferation and differentiation than control, PLLA scaffolds. Fibrous morphology of the scaffold had a pronounced impact on the morphology of the cells in direct contact with the scaffold surface, but not on cell proliferation and differentiation. (Peng et al., 2011) ${ }^{28}$

Fujihara et al. and Seol et al. prepared PCL/Ca composite scaffolds.(Erisken et al., 2008; Fujihara et al., 2005; Ko et al., 2008) ${ }^{29}$ Their results showed that these worked well as scaffolds and they discussed the impact of $\mathrm{Ca}$ ions, which are known to act as nucleation sites, as a promoter for the formation of low crystalline carbonate apatite crystals when released.(Figure 8).

Lee et al. investigated several PLGA/gelatin/HAp composite nanofibers and proved the applicable properties of these composites for bone tissue engineering. In this study, gelatin was shown to have good-interaction with cells when included with HAp as an electrospun fiber. They generated several nanofibrous PLGA/gelatin/HAp composites having various PLGA and gelatin blend ratios and they characterized the physical properties and morphologies of these nanofibrous composites. [Table 1] (Figure 9)

These composites showed different properties including cell adhesion and proliferation ability. (Figure 10) The results suggest that blending of gelatin and HAp with PLGA electrospun nanofibrous composites provides a good environment for bone regeneration. An optimal blend was found to occur at 3:7 of PLGA and gelatin. These composites with enhanced HAp exposure on the nanofiber surface, not only supported and provided sufficient adhesion for growth of MC3T3, osteoblast-like cells but also have properties desirable for use as a bone tissue scaffolds. (Lee et al., 2010) ${ }^{30}$

(A)

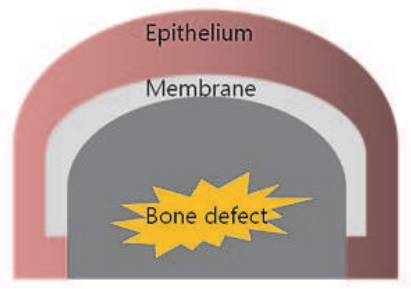

(B)

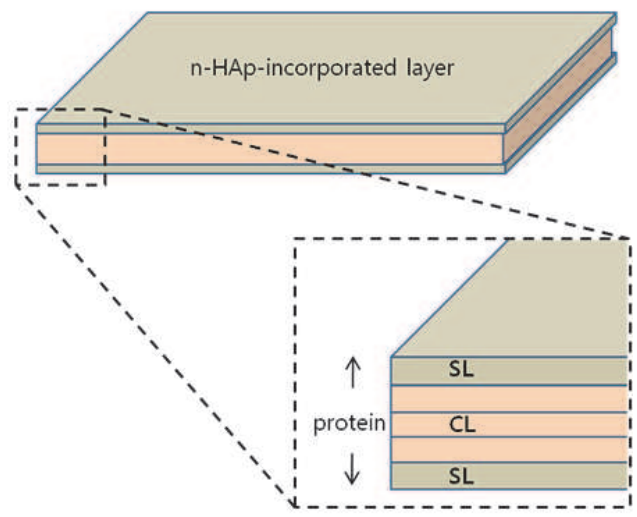

Fig. 8. Structural illustration of the functionally graded periodontal membrane(FGM). (A) Transversal illustration in guided bone regeneration. (B) Details of the core layer (CL) and the surfaces(SLs) morphology. 


\begin{tabular}{c|cccc}
$\begin{array}{c}\text { Mass ratio } \\
(\text { PLGA }: \text { Gelatin }: \text { HAp) }\end{array}$ & $\begin{array}{c}\text { Mean } \\
\text { Diameter( } \mu \mathrm{m})\end{array}$ & $\begin{array}{c}\text { Ultimate Tensile } \\
\text { Stress(MPa) }\end{array}$ & $\begin{array}{c}\text { Elongationat } \\
\text { Break Point }(\%)\end{array}$ & $\begin{array}{c}\text { Young's Modulus } \\
(\mathrm{Mpa})\end{array}$ \\
\hline $100: 0: 20$ & $0.27 \pm 0.08$ & $2.6 \pm 0.2$ & $22.5 \pm 4.0$ & $86.7 \pm 8.6$ \\
\hline $70: 30: 20$ & $0.38 \pm 0.14$ & $3.3 \pm 0.2$ & $26.6 \pm 9.3$ & $79.8 \pm 5.9$ \\
\hline $30: 70: 20$ & $0.53 \pm 0.17$ & $4.0 \pm 0.1$ & $4.1 \pm 0.7$ & $294.8 \pm 14.5$ \\
\hline $0: 100: 20$ & $1.65 \pm 0.13$ & $1.8 \pm 0.1$ & $2.2 \pm 0.8$ & $142.1 \pm 14.2$ \\
\hline
\end{tabular}

Table 1. Physical properties of PLGA/gelatin/HAp nanofibrous composites as various blending ratios.
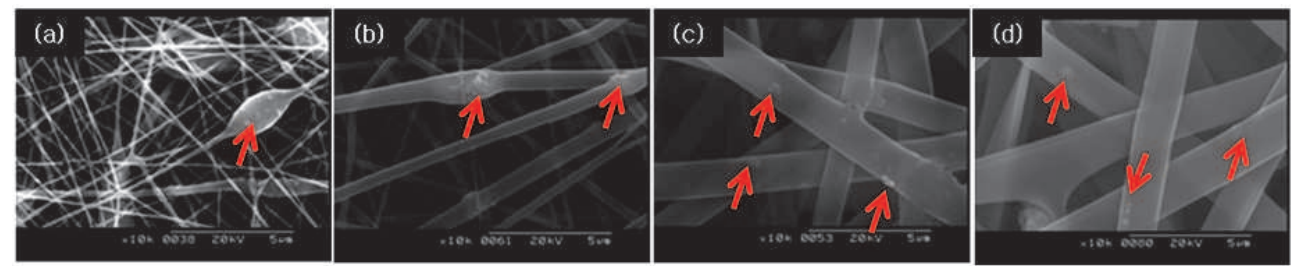

Fig. 9. Scanning electron microscopy and dispersed HAp composites. (a) PGH-1, (b) PGH-2, (c) PGH-3, (d) PGH-4.
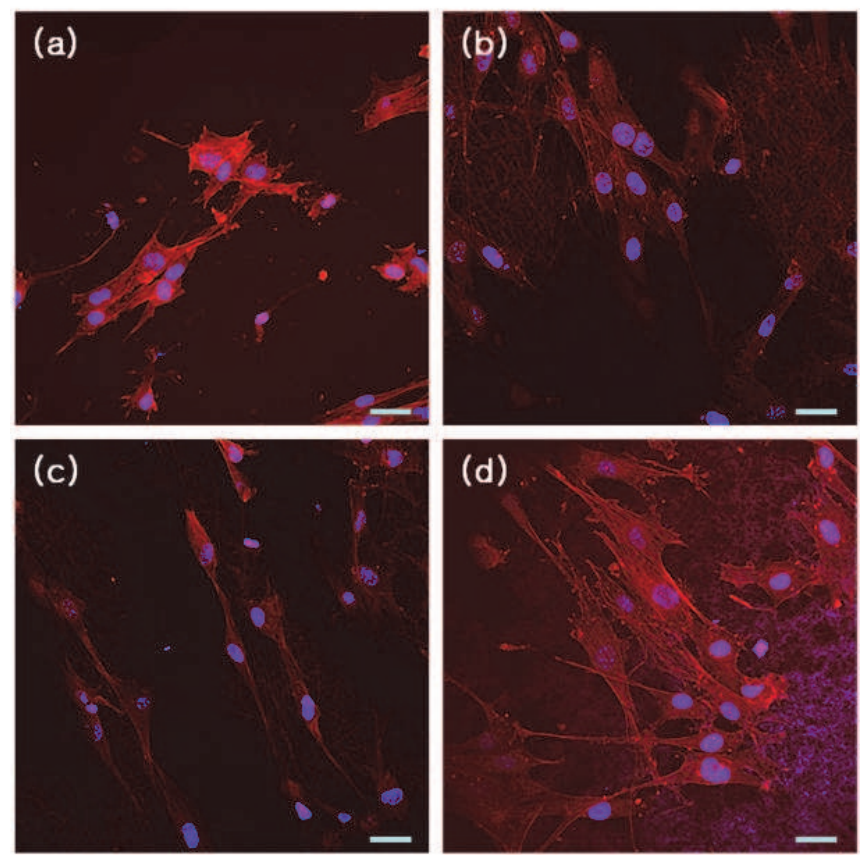

Fig. 10. Immunofluorescence of F-actin filaments in osteoblast-like cell(MC3T3) on nanofibrous PLGA/gelatin/HAp composites; (a) PGH-1, (b) PGH-2, (c) PGH-3, (d) PGH-4. Scale bars indicated $50 \mu \mathrm{m}$. 


\subsubsection{Non-components of natural bone}

Kim et al. developed a nanocomposite made up of a bioactive glass in a nanofibrous form of PLA. (Figure 11)

Bioactive glasses were uniformly dispersed into the PLA nanofibers The nanocomposites induced rapid formation of a hydroxy carbonate apatite layer on the surface under simulated physiological medium. As the amount of bioactive nanofiber increased, the in vitro bioactivity of the nanocomposite was improved. The osteoblast response to the nanocomposites were assessed in terms of cell proliferation, differentiation, and mineralization. Osteoblasts attached and grew well on the nanocomposites and secreted collagenous proteins during the initialculturing periods. Herein the differentiation of cells and mineralized products of the composite nanofiber were observed to be significantly higher than that of pure PLA. (Kim et al., 2008) $)^{31}$

Multiwalled carbon nanotubes (MWCNTs) and demineralized bone powders (DBPs) have also been used as composites with scaffolds for bone tissue engineering. (Shao et al., 2011; Ko et al., 2008) $)^{32}$

Electrospinning

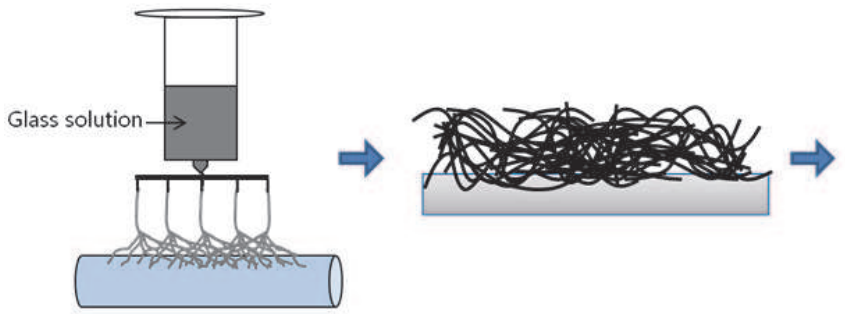

Hydridizing

Thermal pressing

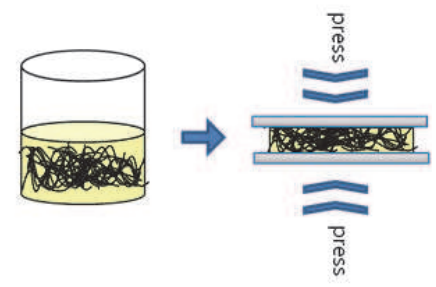

Fig. 11. Schematic illustration of the processing introduced to fabricate bioactive glass nanofiber and its nanocomposite result with PLA.

\subsection{Co-ELSP with inorganic compounds}

Generally, collagen accumulation, mineralization and osteoblast-specific gene expressions are also increased on electrospun scaffolds. Furthermore, some have fabricated functionalized electrospun scaffolds by using biomolecules, including calcium phosphate (Ca-P), HAp, and simulated body fluids (SBF). Rodriguez et al. generated a scaffold which mimicked bone structure and chemical composition using Ca-P and cellulose. As far as we know, most Ca composite have a similar chemical composition to the mineral constituents of bones. They obtained the cellulose fibrous scaffold by the electrospinning method, and the surface was modified with SBF and carboxymethyl cellulose (CMC) adsorption. They also investigated the bioactivity of electrospun regenerated cellulose scaffolds under physiological conditions and evaluated the enhancement of the biomineralization process due to $\mathrm{CMC}$ adsorption on these regenerated cellulose (RC) fibers. The biomimetic 
properties of Ca-P crystals on electrospun fibers were enhanced by the carboxyl groups added with CMC. This enhanced Ca-P mineralization on cellulose scaffolds can be accomplished by increasing the negative charge and by heating. Furthermore mineralization with SBF solution indicated that cellulose scaffolds can be a good candidate for use as a bioactive substrate for bone tissue engineering applications. (Rodriguez et al., 2011) ${ }^{33}$ Rusu et al. produced HAp and chitosan hybrid fibrous composites using the electrospinning method. Pure chitosan is difficult to electrospin due to limited solubility, high viscosity, dielectric constant, and three-dimensional networks of strong hydrogen bonds. Owing to these poor electrospinnability and the adverse effects of the non-electrospinnable HAp nanoparticles and their aggregates, there have been very few attempts of using HA and chitosan for bone tissue engineering. (Rusu et al., 2005) (34 $^{34}$

\subsection{Other treatment}

\subsubsection{Drug loading}

Electrospun fibrous scaffolds have been proposed as drug release substrates by exploiting the direct dependence of the release rate of the drug deposited on the surface or imbedded in the fiber.

An osteogenic differentiation factor, dexamethasone (DEX), was incorporated into electrospun PCL nanofibers by Martins et al. The DEX incorporated into the polymeric carrier is in amorphous state and does not influence the typical nanofibers morphology. They studied in vitro drug released behavior and expression of osteogenic activity of the loaded growth/differentiation factor using human bone marrow mesenchymal stem cell. Overall, this data proposed that scaffold can be used as relevant drug carriers for bone tissue engineering strategies. (Martins et al., 2010) ${ }^{35}$

\subsubsection{Hybrid scaffold fabricated by ELSP and other method}

Erisken et al. demonstrate the fabrication and utilization of functionally graded non woven meshes of PCL incorporated with various concentrations of TCP nanoparticles using a hybrid twin-screw extrusion/electrospinning (TSEE) process. TSEE process allows various solid and liquid ingredients and their melting, dispersion, and pressurization together with electrospinning within the confines of a single process. They demonstrated in vitro that the ability to incorporate the $\beta$-TCP nanoparticles into PCL nanofibers in a controlled manner enabled better mimicking of the composition and structure of natural bone tissue. (Erisken et al., 2008) 36

\subsubsection{Conjugation with RGD}

In 2010, poly(L-lactide) fiber meshes coated with a poly(lactide-co-ethylene oxide furmarate) (PLEOF) conjugated with Arg-Gly-Asp (RGD) peptide were reported by Xu et al. (Figure 12) Their scaffold is a fiber-reinforced hydrogel/HA laminate composite. Laminates of electrospun PLLA fiber mesh were synthesized. In this report, acrylamide-terminated RGD peptide (Ac-RGD) conjugated to the PLEOF/HA hydrogel phase could promote focal points of adhesion and lamination which increases the extent of mineralization of bone marrow stromal (BMS) cells and this resulted in significantly higher expression of osteopontin and osteocalcin as compared to the hydrogel or laminates without HAp or RGD. (Xu et al., 2010) $)^{37}$ 


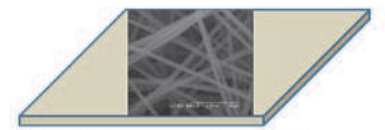

(a) Electrospun fiber mesh

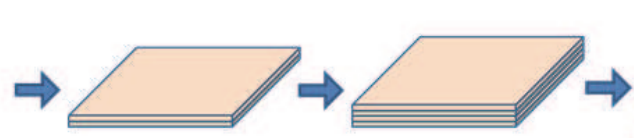

(c) lamination

(d) multi-layer laminate

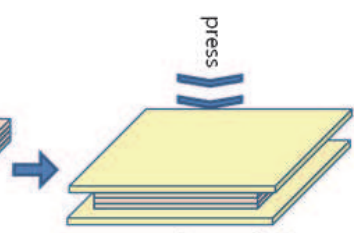

(e) Press and cross linking

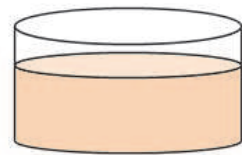

(b) Hydrogel precursor solution

Fig. 12. Schematic diagram for the fabrication procedure of fiber-reinforced hydrogel/HA laminated composite. (a) The electrospun PLLA nanofiber mesh was placed on a Teflon plate, (b) the hydrogel precursor solution was brushed over the fibers, and (c) another fiber layer was placed on top of the precursor solution. This process was repeated to produce a multi-layer laminated composite (d). The precursor solution acted as a "glue" to hold together the fiber layers. Then, another Teflon plate was placed on top of the laminate (e), a pressure of $4.7 \mathrm{kPa}$ was applied to the assembly to squeeze out the extra solution and it was allowed to crosslink (e). The hydrogel/HA precursor solution was prepared by mixing HA nanocrystals, BISAM crosslinker, Ac-GRGD cell adhesion peptide, APS initiator and TMEDA accelerator in PBS.

\subsubsection{Fabrication of 3D fibrous mesh-form scaffolds using electrospun fibrous sheet}

Mimicking the morphology of the natural extracellular matrix is considered a promising method for generating scaffolds for tissue engineering. Martins et al. developed hierarchical starch-based scaffolds which were fabricated by a combination of starch-polycaprolactone micro- and polycaprolactone nano-motifs. These scaffolds were produced by rapid prototyping (RP) and electrospinning method, respectively. The bulk of the composed/hierarchical scaffolds had enhanced cell retention and hierarchical scaffolds displayed significantly improved cell proliferation and osteoblastic activity, as assessed by alkaline phosphatase quantification. These observations support their hypothesis that the integration of nanofibers into 3D RP scaffolds substantially improves biological performance for use in bone tissue engineering. (Martins et al., 2009) $)^{38}$

\section{Conclusion}

The extracellular matrix (ECM) plays an important role in the function of bone growth. The major organic components of bone matrix are woven collagenous fibrils within hydrated polysaccharide chains such as hydroxyapatite (HAp), acting efficiently in response to external stress, and transmitting signals between cells and external stimulus via intracellular signaling cascades. Therefore, there has been a great deal of research in terms of type of materials, structural designs, and biofunctions which best mimic ECM using the electrospinning method. 
Advances in nanotechnology have significantly impacted bone tissue engineering and bone regeneration. By electrospinning method, nano and micrometer-sized entities, structural and surface morphologies have been developed with unexpected properties, which can be used with various materials.

Development of more effective scaffold by means of electrospinning with the aim to aid in bone regeneration is in progress. Early scaffolds for bone tissue engineering were fabricated using electrospun polymers only. However the scaffolds provided not only mimic the ECM histoarchitecture but biological and mechanical support was needed at the same time during the healing process. This exerts a modulatory effect on cell colonization and orchestrates the bone regeneration response in a regulated manner. Inorganic particles implanted in the matrix exhibit high mechanical strength and good biocompatibility. Therefore in many studies Hap is widely used because it is similar to natural bone components and enhances the mechanical properties of scaffold. Finally nanofibers, contained drug or growth factor, can support cell growth and differentiation while at the same time maintaining physical structure.

The latest, in bone tissue engineering is the use of appropriate biomaterials including inorganic natural bone compounds, inorganic components to enhanced mechanical properties, and natural polymers. A number of studies have been done and are in progress in order to perfect the process of bone tissue regeneration.

\section{Acknowledgment}

This work was supported by the grant from the Ministry of Knowledge Economy of Korea (10035291), the Ministry of Education, Science and Technology of Korea (20110001689), and the Korea Science \& Engineering Foundation (20100002088).

\section{References}

[1] Feng, X. \& McDonald, M. (2011). Disorders of bone remodeling. Annu Rev Pathol, 6, 121145.

[2] Seong, J. M., Kim, B. -C., Park, J. -H., Kwon, I. K., Mantalaris, A. \& Hwang, Y. -S. (2010). Stem cells in bone tissue engineering. Biomed Mater, 5, 062001

[3] Parfitt, A. M. (1994). Osteonal and hemi-osteonal remodeling: the spatial and temporal framework for signal traffic in adult human bone. J Cell Biochem, 55, 273-286

Seeman, E. (2009). Bone modeling and remodeling. Crit Rev Eukaryot Gene Expr, 19, 219 233

[4] Yasuda, H., Shima, N., Nakagawa, N., Yamaguchi, K., Kinosaki, M., Mochizuki, S.-I., Tomoyasu, A., Yano, K., Goto, M., Murakami, A., Tsuda, E., Morinaga, T., Higashio, K., Udagawa, N., Takahashi, N. \& Suda, T. (1998). Osteoclast differentiation factor is a ligand for osteoprotegerin/osteoclastogenesis inhibitory factor and is identical to TRANCE/RANKL. Proc Natl Acad Sci, 95, 3597-3602

Lacey, D. L., Timms, E., Tan, H. -L., Kelley, M. J., Dunstan, C. R., Burgess, T., Elliott, R., Colombero, A., Elliott, G., Scully, S., Hsu, H., Sullivan, J., Hawkins, N., Davy, E., Capparelli, C., Eli, A., Qian, Y. -X., Kaufman, S., Sarosi, I., Shalhoub, V., Senaldi, G., Guo, J., Delaney, J. \& Boyle, W. J. (1998). Osteoprotegerin ligand is a cytokine that regulates osteoclast differentiation and activation. Cell, 93, 165-176

[5] Nakagawa, N., Kinosaki1, M., Yamaguchi, K., Shima, N., Yasuda, H., Yano, K., Morinaga, T. \& Higashio, K. (1998). RANK is the essential signaling receptor for 
osteoclast differentiation factor in osteoclastogenesis. Biochem Biophys Res Commun, 253, 395-400

Hsu, H., Lacey, D. L., Dunstan, C. R., Solovyev, I., Colombero, A., Timms, E., Tan, H. L., Elliott, G., Kelley, M. J., Sarosi, I., Wang, L., Xia, X. -Z., Elliott, R., Chiu, L., Black, T., Scully, S., Capparelli, C., Morony, S., Shimamoto, G., Bass, M. B. \& Boyle, W. J. (1999). Tumor necrosis factor receptor family member RANK mediates osteoclast differentiation and activation induced by osteoprotegerin ligand. Proc Natl Acad Sci, 96, 3540-3545

[6] Newby, A.C. (2008). Metalloproteinase expression in monocytes and macrophages and its relationship to atherosclerotic plaque instability. Arterioscler Thromb Vasc Biol, 28, 2108-2114

[7] Yun, Y. P., Kim, S. E., Lee, J. B., Heo, D. N., Bae, M. S., Shin, D. -R., Lim, S. -B., Choi, K. K., Park, S. J. \& Kwon, I. K. (2009). Comparison of osteogenic differevtiation from adipose-derived stem cells, mesenchymal stem cells, and pulp cells on PLGA/Hydroxyapatite nanofiber. Tissue Engineering and Regenerative Medicine, 6, 336-345

Seyedjafari, E., Soleimani, M., Ghaemi, N. \& Shabani, I. (2010). Nanohydroxyapatitecoated electrospun poly(L-lactide) nanofibers enhance osteogenic differentiation of stem cells and induce ectopic bone formation. Biomacromolecules, 11, 3118-3125

Haslauer, C. M., Moghe, A. K., Osborne, J. A., Gupta, B. S. \& Loboa, E. G. (2011). Collagen-PCL sheath-core bicomponent electrospun scaffolds increase osteogenic differentiation and calcium accretion of human adipose-derived stem cells, J Biomater Sci, Vol 22, 13, 1695-1712

Lee, H., Yeo, M., Ahn, S. H., Kang, D. -O., Jang, C. H., Lee, H., Park, G. -M. \& Kim, G. H. (2011). Designed hybrid scaffolds consisting of polycaprolactone microstrands and electrospun collagen-nanofibers for bone tissue regeneration, J Biomed Mater Res Part B: Appl Biomater, 97B, 263-270

Burg, K. J. L., Porter, S. \& Kellam, J. F. (2000). Biomaterial developments for bone tissue engineering, Biomater, 21, 2347-2359

[8] Yoshimoto, H., Shin, Y. M., Terai, H. \& Vacanti, J. P. (2003). A biodegradable nanofiber scaffold by electrospinning and its potential for bone tissue engineering, Biomater, 24(12), 2077-2082

[9] Erisken, C., Kalyon, D. M. \& Wang, H. (2008). Funtionally graded electrospun polycaprolactone and $\beta$-tricalcium phosphate nanocomposites for tissue engineering applications, Biomater, 29, 4065-4073

Fujihara, K., Kotaki, M. \& Ramakrishna, S. (2005). Guided bone regeneration membrane made of polycaprolactone/calcium carbonate composite nano-fibers, Biomater, 26, 4139-4147

Seol, Y. -J., Kim, K. -H., Kim, I. A. \& Rhee, S. -H.(2010). Osteoconductive and degradable electrospun nonwoven poly( $\varepsilon$-caprolactone)/CaO-SiO2 gel composite fabric, $J$ Biomed Mater Res Part A, 94A, 649-659

Kolambkar, Y. M., Peister, A., Ekaputra, A. K., Hutmacher, D. W. \& Guldberg, R. E. (2010). Colonization and Osteogenic Differentiation of Different Stem Cell Sources on Electrospun Nanofiber Meshes, Tissue Eng Part A, 16(10), 3219-3230

[10] Kolambkar, Y. M., Peister, A., Ekaputra, A. K., Hutmacher, D. W. \& Guldberg, R. E. (2010). Colonization and Osteogenic Differentiation of Different Stem Cell Sources on Electrospun Nanofiber Meshes, Tissue Eng Part A, 16(10), 3219-3230

[11] Cho, W. J., Kim, J. H., Oh, S. H., Nam, H. H., Kim, J. M. \& Lee, J. H. (2009). Hydrophilized polycaprolactone nanofiber mesh-embedded poly(glycolic-co-lactic 
acid) membrane for effective guided bone regeneration, J Biomed Mater Res, 91A, 400-407

[12] Badami, A. S., Kreke, M. R., Thompson, M. S., Riffle, J. S. \& Goldstein, A. S. (2006). Effect of fiber diameter on spreading, proliferation, and differentiation of osteoblastic cells on electrospun poly(lactic acid) substrates, Biomater, 27, 596-606

[13] Bashur, C. A., Shaffer, R. D., Dahlgren, L. A., Guelcher, S. A. \& Goldstein, A. S. (2009). Effect of fiber diameter and alignment of electrospun polyurethane meshes on mesenchymal progenitor cells, Tissue Eng: Part A, 15(9), 2435-2445

[14] Haslauer, C. M., Moghe, A. K., Osborne, J. A., Gupta, B. S. \& Loboa, E. G. (2011). Collagen-PCL sheath-core bicomponent electrospun scaffolds increase osteogenic differentiation and calcium accretion of human adipose-derived stem cells, $J$ Biomater Sci, Vol 22, 13, 1695-1712

[15] Haslauer, C. M., Moghe, A. K., Osborne, J. A., Gupta, B. S. \& Loboa, E. G. (2011). Collagen-PCL sheath-core bicomponent electrospun scaffolds increase osteogenic differentiation and calcium accretion of human adipose-derived stem cells, $J$ Biomater Sci, Vol 22, 13, 1695-1712

Lee, H., Yeo, M., Ahn, S. H., Kang, D. -O., Jang, C. H., Lee, H., Park, G. -M. \& Kim, G. H. (2011). Designed hybrid scaffolds consisting of polycaprolactone microstrands and electrospun collagen-nanofibers for bone tissue regeneration, J Biomed Mater Res Part B: Appl Biomater, 97B, 263-270

O'Brien, F. J., Harley, B. A., Yannas, I. V. \& Gibson, L. (2004). Influence of freezing rate on pore structure in freeze dried collagen GAG scaffolds, Biomater, 25, 1077-1086

Matthews, J. A., Wnek, G. E., Simpson, D. G. \& Bowlin, G. L. (2002). Electrospinning of collagen nanofibers, Biomacromol, 3, 232-238

Beachley, V. \& Wen, X. (2010). Polymer nanofibrous structures: Fabrication, biofunctionalization, and cell interactions, Prog Polym Sci, 35, 868-892

[16] Kim, K. -H., Jeong, L., Park, H. -N., Shin, S. -Y., Park, W. -H., Lee, S. -C., Kim, T. -I., Park, Y. -J., Seol, Y. -J., Lee, Y. -M., Ku, Y., Rhyu, I. -C., Han, S. -B. \& Chung, C. -P. (2005). Biological efficacy of silk fibroin nanofiber membranes for guided bone regeneration, J Biotech, Vol. 120, 327-339

[17] Haslauer, C. M., Moghe, A. K., Osborne, J. A., Gupta, B. S. \& Loboa, E. G. (2011). Collagen-PCL sheath-core bicomponent electrospun scaffolds increase osteogenic differentiation and calcium accretion of human adipose-derived stem cells, J Biomater Sci, Vol 22, 13, 1695-1712

Lee, H., Yeo, M., Ahn, S. H., Kang, D. -O., Jang, C. H., Lee, H., Park, G. -M. \& Kim, G. H. (2011). Designed hybrid scaffolds consisting of polycaprolactone microstrands and electrospun collagen-nanofibers for bone tissue regeneration, J Biomed Mater Res Part B: Appl Biomater, 97B, 263-270

Bottino, M. C., Thomas, V. \& Janowski, G. M.(2011). A novel spatially designed and functionally graded electrospun membrane for periodontal regeneration, Acta Biomater, Vol. 7, 216-224

[18] Haslauer, C. M., Moghe, A. K., Osborne, J. A., Gupta, B. S. \& Loboa, E. G. (2011). Collagen-PCL sheath-core bicomponent electrospun scaffolds increase osteogenic differentiation and calcium accretion of human adipose-derived stem cells, $J$ Biomater Sci, Vol 22, 13, 1695-1712

[19] Erisken, C., Kalyon, D. M. \& Wang, H. (2008). Funtionally graded electrospun polycaprolactone and $\beta$-tricalcium phosphate nanocomposites for tissue engineering applications, Biomater, 29, 4065-4073 
Fujihara, K., Kotaki, M. \& Ramakrishna, S. (2005). Guided bone regeneration membrane made of polycaprolactone/calcium carbonate composite nano-fibers, Biomater, 26, 4139-4147

Seol, Y. -J., Kim, K. -H., Kang, Y. M., Kim, I. A. \& Rhee, S. -H. (2009). Bioactivity, preosteoblastic cell responses, and osteoconductivity evaluations of the electrospun non-woven SiO2-CaO gel fabrics, J Biomed Mater Res Part B: Appl Biomater, 90B, 679687

Seol, Y. -J., Kim, K. -H., Kim, I. A. \& Rhee, S. -H.(2010). Osteoconductive and degradable electrospun nonwoven poly( $\varepsilon$-caprolactone)/CaO-SiO2 gel composite fabric, J Biomed Mater Res Part A, 94A, 649-659

Ko, E. K., Jeong, S. I., Rim, N. G., Lee, Y. M., Shin, H. \& Lee, B. -K. (2008). In vitro osteogenic differentiation of human mesenchymal stem cells and in vivo bone formation in composite nanofiber meshes, Tissue Eng Part A, Vol. 14, No. 12, 21052119

[20] Seyedjafari, E., Soleimani, M., Ghaemi, N. \& Shabani, I. (2010). Nanohydroxyapatitecoated electrospun poly(L-lactide) nanofibers enhance osteogenic differentiation of stem cells and induce ectopic bone formation. Biomacromolecules, 11, 3118-3125

Nie, H. \& Wang, C. -H. (2007). Fabrication and characterization of PLGA/HAp composite scaffolds for delivery of BMP-2 plasmid DNA, J Control Release, Vol. 120, 111-121

Mouthuy, P. -A., Ye, H., Triffitt, J., Oommen, G. and Cui, Z. (2010). Physico-chemical characterization of functional electrospun scaffolds for bone and cartilage tissue engineering, Proc IMechE, Vol. 224, No. 12, 1401-1414

[21] Nie, H. \& Wang, C. -H. (2007). Fabrication and characterization of PLGA/HAp composite scaffolds for delivery of BMP-2 plasmid DNA, J Control Release, Vol. 120, 111-121

Mouthuy, P. -A., Ye, H., Triffitt, J., Oommen, G. and Cui, Z. (2010). Physico-chemical characterization of functional electrospun scaffolds for bone and cartilage tissue engineering, Proc IMechE, Vol. 224, No. 12, 1401-1414

Peng, F., Yu, X. \& Wei, M. (2011). In vitro cell performance on hydroxyapatite particles/poly(L-lactic acid) nanofibrous scaffolds with an excellent particle along nanofiber orientation, Acta Biomater, Vol. 7, No. 6, 2585-2592

[22] Bottino, M. C., Thomas, V. \& Janowski, G. M.(2011). A novel spatially designed and functionally graded electrospun membrane for periodontal regeneration, Acta Biomater, Vol. 7, 216-224

[23] Seyedjafari, E., Soleimani, M., Ghaemi, N. \& Shabani, I. (2010). Nanohydroxyapatitecoated electrospun poly(L-lactide) nanofibers enhance osteogenic differentiation of stem cells and induce ectopic bone formation. Biomacromolecules, 11, 3118-3125

[24] Whited, B. M., Whitney, J. R., Hofmann, M. C., Xu, Y. \& Rylander, M. N. (2011). Preosteoblast infiltration and differentiation in highly porous apatite-coated PLLA electrospun scaffolds, Biomater, Vol. 32, No. 9, 2294-2304

[25] Rainer, A., Spadaccio, C., Sedati, P., Marco, F. D., Carotti, S., Lusini, M., Vadala, G., Martino, A. D., Morini, S., Chello, M., Covino, E., Denaro, V. \& Trombetta, M. (2011). Electrospun hydroxyapatite-functionalized PLLA scaffold: Potenial applications in sternal bone healing, Ann Biomed Eng, Vol. 39, No. 7, 1882-1890

[26] Jose, M. V., Thomas, V., Johnson, K. T., Dean, D. R. \& Nyairo, E. (2009). Aligned PLGA/HA nanofibrous nanocomposite scaffolds for bone tissue engineering, Acta Biomater, 5, 305-315 
[27] Yun, Y. P., Kim, S. E., Lee, J. B., Heo, D. N., Bae, M. S., Shin, D. -R., Lim, S. -B., Choi, K. K., Park, S. J. \& Kwon, I. K. (2009). Comparison of osteogenic differevtiation from adipose-derived stem cells, mesenchymal stem cells, and pulp cells on PLGA/Hydroxyapatite nanofiber. Tissue Engineering and Regenerative Medicine, 6, 336-345

[28] Peng, F., Yu, X. \& Wei, M. (2011). In vitro cell performance on hydroxyapatite particles/poly(L-lactic acid) nanofibrous scaffolds with an excellent particle along nanofiber orientation, Acta Biomater, Vol. 7, No. 6, 2585-2592

[29] Erisken, C., Kalyon, D. M. \& Wang, H. (2008). Funtionally graded electrospun polycaprolactone and $\beta$-tricalcium phosphate nanocomposites for tissue engineering applications, Biomater, 29, 4065-4073

Fujihara, K., Kotaki, M. \& Ramakrishna, S. (2005). Guided bone regeneration membrane made of polycaprolactone/calcium carbonate composite nano-fibers, Biomater, 26, 4139-4147

Ko, E. K., Jeong, S. I., Rim, N. G., Lee, Y. M., Shin, H. \& Lee, B. -K. (2008). In vitro osteogenic differentiation of human mesenchymal stem cells and in vivo bone formation in composite nanofiber meshes, Tissue Eng Part A, Vol. 14, No. 12, 21052119

[30] Lee, J. B., Kim, S. E., Heo, D. N. \& Kwon, I. K. (2010). In vitro characterization of nanofibrous PLGA/gelatin/Hydroxyapatite composite for bone tissue engineering, Macromol Res, Vol. 18, No. 12, 1195-1202

[31] Kim, H.-W., Lee, H. -H. \& Chun, G. -S. (2008). Bioactivity and osteoblast responses of novel biomedical nanocomposites of bioactive glass nanofiber filled poly(lactic acid), J Biomed Mater Res, 85A, 651-663

[32] Shao, S., Zhou, S., Li, L., Li, J., Luo, C., Wang, J., Li, X. and Weng, J. (2011). Osteoblast function on electrically conductive electrospun PLA/MWCNTs nanofiber, Biomater, $32,2821-2833$

Ko, E. K., Jeong, S. I., Rim, N. G., Lee, Y. M., Shin, H. \& Lee, B. -K. (2008). In vitro osteogenic differentiation of human mesenchymal stem cells and in vivo bone formation in composite nanofiber meshes, Tissue Eng Part A, Vol. 14, No. 12, 21052119

[33] Rodriguez, K., Renneckar, S. and Gatenholm, Paul. (2011). Biomimetic calcium phosphate crystal mineralization on electrospun cellulose-based scaffolds, ACS Appl Mater Interfaces, 3, 681-689

[34] Rusu, V. M., Ng, C. -H., Wilke, M., Tiersch, B., Fratzl, P. \& Peter, M. G. (2005). Sizecontrolled hydroxyapatite nanoparticles as self-organized organic-inorganic composite materials, Biomater, 26, 5414-5426

[35] Martins, A., Duarte, A. R. C., Faria, S., Marques, A. P., Reis, R. L. \& Neves, N. M. (2010). Osteogenic inductions of hBMSCs by electrospun scaffolds with dexamethasone release functionality, Biomater, 31, 5875-5885

[36] Erisken, C., Kalyon, D. M. \& Wang, H. (2008). Funtionally graded electrospun polycaprolactone and $\beta$-tricalcium phosphate nanocomposites for tissue engineering applications, Biomater, 29, 4065-4073

[37] Xu, W., Ma, J. \& Jabbari, E. (2010). Material properties and osteogenic differentiation of marrow stromal cells in fiber-reinforced laminated hydrogel nanocomposites, Acta Biomater, 6, 1992-2002

[38] Martins, A., Chung, S., Pedro, A. J., Sousa, R. A., Marques, A. P., Reis, R. L. \& Neves, N. M. (2009). Hierarchical starch-based fibrous scaffold for bone tissue engineering applications, J Tissue Eng Regen Med, 3, 37-42 


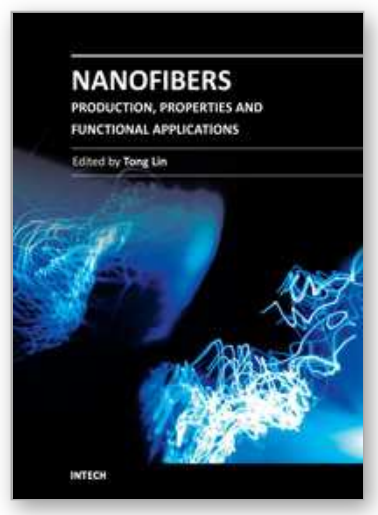

\author{
Nanofibers - Production, Properties and Functional Applications \\ Edited by Dr. Tong Lin
}

ISBN 978-953-307-420-7

Hard cover, 458 pages

Publisher InTech

Published online 14, November, 2011

Published in print edition November, 2011

As an important one-dimensional nanomaterial, nanofibers have extremely high specific surface area because of their small diameters, and nanofiber membranes are highly porous with excellent pore interconnectivity. These unique characteristics plus the functionalities from the materials themselves impart nanofibers with a number of novel properties for advanced applications. This book is a compilation of contributions made by experts who specialize in nanofibers. It provides an up-to-date coverage of in nanofiber preparation, properties and functional applications. I am deeply appreciative of all the authors and have no doubt that their contribution will be a useful resource for anyone associated with the discipline of nanofibers.

\title{
How to reference
}

In order to correctly reference this scholarly work, feel free to copy and paste the following:

Ha Na Park, Jung Bok Lee, Ho-Jin Moon, Dae Hyeok Yang and II Keun Kwon (2011). The Potential of Biomimetic Electrospun-Nanofibrous Scaffolds for Bone Tissue Engineering, Nanofibers - Production, Properties and Functional Applications, Dr. Tong Lin (Ed.), ISBN: 978-953-307-420-7, InTech, Available from: http://www.intechopen.com/books/nanofibers-production-properties-and-functional-applications/the-potentialof-biomimetic-electrospun-nanofibrous-scaffolds-for-bone-tissue-engineering

\section{INTECH}

open science | open minds

\section{InTech Europe}

University Campus STeP Ri

Slavka Krautzeka 83/A

51000 Rijeka, Croatia

Phone: +385 (51) 770447

Fax: +385 (51) 686166

www.intechopen.com

\section{InTech China}

Unit 405, Office Block, Hotel Equatorial Shanghai

No.65, Yan An Road (West), Shanghai, 200040, China

中国上海市延安西路65号上海国际贵都大饭店办公楼405单元

Phone: +86-21-62489820

Fax: $+86-21-62489821$ 
(C) 2011 The Author(s). Licensee IntechOpen. This is an open access article distributed under the terms of the Creative Commons Attribution 3.0 License, which permits unrestricted use, distribution, and reproduction in any medium, provided the original work is properly cited. 